\title{
Artículo
}

\section{Interfaz visual para recuperación de información basada en análisis de metadatos, escalamiento multidimensional y efecto ojo de pez}

\author{
Por Yusef Hassan-Montero y Víctor Herrero-Solana
}

Resumen: En el presente trabajo se propone un modelo original de interfaz visual para la recuperación de información o VI$R I$ (visual information retrieval interface), orientada a sitios web descritos por metadatos. Además, se explica un prototipo experimental basado en dicho modelo resultado de la combinación de tres métodos: minería de metadatos, escalamiento multidimensional o MDS (multidimensional scaling), y una técnica de focus+context denominada "ojo de pez". Por último se lleva a cabo un test de usuarios sobre el citado prototipo. Los resultados sugieren que el modelo de VIRI propuesto resulta útil para la exploración y recuperación de información, así como satisfactorio para el usuario final.

Palabras clave: Visualización de información, Minería de datos, Escalamiento multidimensional, Focus+context, Ojo de pez.

Title: Information retrieval interface based on metadata mining, multidimensional scaling and fisheye technique

Abstract: This paper proposes an original model of the visual information retrieval interface (VIRI), aimed at websites described by metadata. The paper also describes the development of an experimental prototype based on the proposed model. The

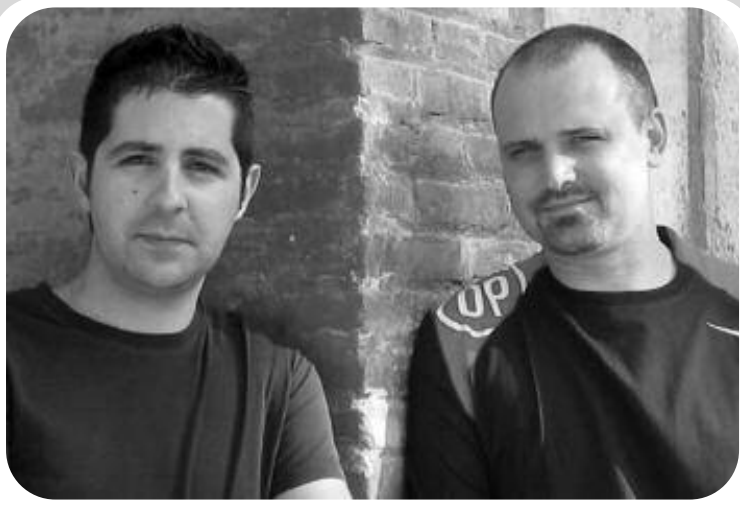

Yusef Hassan Montero es licenciado en biblioteconomía y documentación, e investigador contratado por el grupo SClmago en la Universidad de Granada, con cargo al proyecto Atlas of Science. Sus líneas de investigación se centran en la interacción persona-ordenador, visualización y recuperación de información. Además es co-editor de NoSoloUsabilidad.com, magazín electrónico sobre diseño web centrado en el usuario, usabilidad y arquitectura de la información.

Víctor Herrero-Solana, doctor en documentación. Profesor de la Facultad de Documentación de la Universidad de Granada e investigador del Grupo SClmago.

applied methodology for the prototype's development is a combination of three methods: Metadata mining, Multidimensional scaling (MDS), and a Focus +Context technique called Fisheye. Finally, a user test is conducted, whose results suggest that the proposed model of VIRI is useful for information browsing and retrieval, and is satisfying for the end-user as well.

Keywords: Information visualisation, Data mining, Multidimensional scaling, Focus+context, Fisheye.

Hassan-Montero, Yusef; Herrero-Solana, Víctor. «Interfaz visual para recuperación de información basada en análisis de metadatos, escalamiento multidimensional y efecto ojo de pez». En: El profesional de la información, 2006, julio-agosto, v. 15, n. 4, pp. 278-289.

\section{Introducción}

La world wide web representa en la actualidad el mayor espacio de información existente debido a su constante y exponencial crecimiento. A pesar de que este volumen le confiere un gran valor informativo, conlleva una problemática correlacionada: el incremento para el usuario de la dificultad para encontrar y localizar información relevante que satisfaga sus necesidades de información. No es de extrañar, por tanto, que la investigación en técnicas de recuperación de in- formación (RI) haya experimentado un gran auge a partir del surgimiento de la www.

Los sistemas de RI basados en querying -en los que el usuario debe introducir una serie de términos de búsqueda para que reciba una lista de resultados ordenada por relevancia respecto a la consulta- son los que mayor atención han recibido hasta la fecha por parte de la comunidad científica dedicada a investigar el tema. Este hecho ha originado que actualmente exista multitud de implementaciones prácticas de gran utilidad para el usuario final, siendo el motor de búsqueda 
dio se encuentra estructurado en los siguientes apartados: (2) descripción de un modelo metodológico para la producción de VIRIs; (3) especificación de los objetivos generales y específicos perseguidos; (4) metodología y materiales empleados; (5) presentación de los resultados en forma de prototipo funcional de la interfaz; (6) descripción de los resultados obtenidos tras la evaluación del prototipo mediante un test de usuarios; (7) resumen de las conclusiones y futuras vías de investigación.

\section{Modelo metodológico}

Existen varios trabajos que describen marcos metodológicos para la producción de VIRI: Börner; Chen; Boyack, 2003; Herrero-Solana, 2000 y Marcos-Mora, 2004. En el presente estudio se utilizará el descrito por Herrero-Solana y Hassan-Montero (2006), donde se analizan y comparan los diferentes métodos y algoritmos para la producción de VIRI clasificándolos según la etapa del proceso en la que intervienen:

\section{a. Análisis y transformación de los datos}

Consiste en la indización automática del espacio documental, comúnmente en base al modelo de espacio vectorial propuesto por Salton (1989). El objetivo es permitir tanto la clasificación automática del conjunto documental como el descubrimiento de relaciones estructurales subyacentes. Se utilizan técnicas de minería de datos, ya sea de forma individual o combinada, entre las que podemos distinguir procedimientos de minería de contenido, de estructura y de uso (Baeza-Yates, 2004).

\section{b. Algoritmos de clasificación y distribución vi- sual}

Reduce estructuras de datos multidimensionales a un número de dimensiones comprensibles y perceptibles por el ojo humano (1D, 2D ó 3D). El resultado visual de esta etapa puede ser considerado como la abstracción gráfica de este espacio vectorial. Las técnicas y algoritmos de mayor popularidad son: clustering o análisis de conglomerados (Hartigan, 1975); de poda como el escalamiento de red pathfinder o Pfnets (Schvaneveldt, 1990) y de posicionamiento de los nodos como el de Kamada y Kawai (1989); estadísticas multivariantes tales como el escalamiento multidimensional o MDS (multidimensional scaling) (Kruskal; Wish, 1978); y redes neuronales como el modelo de mapas auto-organizativos o SOM (self-organizing map) (Kohonen; 1989). Todos ellos pueden ser considerados complementarios ya que ofrecen diferentes soluciones para un mismo problema (Herrero-Solana, 2000).

\section{c. Transformación visual}

Tiene su razón de ser cuando la representación gráfica obtenida de la anterior etapa no resulta de fácil exploración debido a su tamaño y complejidad, provocando lo que ha venido a denominarse como "sobrecarga visual". En este caso las posibles técnicas a emplear podemos clasificarlas entre las no orientadas a la distorsión visual (Panning, Zooming y Detail+overview) y las orientadas a la distorsión visual (Focus+context).

El trabajo de Herrero-Solana y Hassan-Montero (2006) concluye con el análisis de qué combinaciones entre las técnicas de la segunda y tercera etapa resultan más adecuadas según su compatibilidad (tabla 1).

\begin{tabular}{|r|c|c|c|}
\hline & MDS & SOM & Pfnets \\
\hline Focus+context & - & $\sqrt{ }$ & - \\
\hline Detail+overview & $\mathrm{X}$ & - & $\sqrt{ }$ \\
\hline Zoom and panning & $\sqrt{ }$ & $\sqrt{ }$ & $\sqrt{ }$ \\
\hline
\end{tabular}

Tabla 1. Leyenda:

$\checkmark$ Combinación adecuada

- Adecuada pero con reservas

$\times$ Combinación inadecuada.

Herrero-Solana; Hassan-Montero, 2006.

\section{Objetivos}

Debido a la cantidad de combinaciones posibles de técnicas, algoritmos y tipos de análisis a emplear en la generación de interfaces visuales para la recuperación de información, es lógico que no todas ellas hayan sido suficientemente exploradas y/o investigadas. Por esta razón, los objetivos generales marcados en el presente trabajo son:

- Proponer un modelo de interfaz visual para recuperación de información en espacios web descritos por metadatos.

-Desarrollar un prototipo funcional en base a este modelo.

-Evaluar el modelo propuesto sobre el prototipo desarrollado.

El modelo de VIRI planteado es resultado de las siguientes técnicas:

-Etapa de análisis y transformación de los datos: minería de meta-contenido mediante el análisis de la co-ocurrencia de palabras clave en el conjunto documental.

-Etapa de aplicación de los algoritmos de clasificación y distribución visual: aplicación de $M D S$ para la visualización del espacio documental a través de la representación bidimensional de las palabras clave más representativas. 
-Etapa de técnicas de transformación visual: aplicación de interactividad a la representación, así como de la distorsión visual ojo de pez (fisheye), con el objetivo de facilitar la exploración visual de la interfaz.

El modelo presentado no pretende ser únicamente original por la combinación de técnicas propuesta, sino también en cuanto al método elegido para su aplicación. Por ello, en el presente trabajo se marcaron los siguientes objetivos específicos:

-Exponer un método de análisis de meta-contenido que permita determinar el grado en que cada documento es representado por cada palabra clave, así como cuáles son las más representativas del conjunto documental.

-Desarrollar un método de aplicación del efecto de ojo de pez para representaciones visuales de dispersión.

\section{Métodos y materiales}

\subsection{Muestra documental}

Consta de 269 documentos obtenidos del sitio web Useit, pertenecientes a su sección alertbox y publicados entre junio de 1995 y junio de 2003. La temática general del conjunto documental está referida al estudio de la usabilidad, la interacción persona-ordenador (IPO) y el diseño web, áreas en las que su autor (Jakob Nielsen) es un reconocido investigador. Todos los documentos se encuentran en formato html y tienen aplicados aquellos metadatos básicos para la descripción de su contenido que este lenguaje de marcado permite: título, palabras clave y resumen.

http://www.useit.com

\subsection{Vectorización y análisis de meta-contenido}

En esta etapa se extrae, representa y analiza la meta-información que en la muestra documental se describe mediante etiquetas html para metadatos. Como se demuestra en estudios sobre el uso de metadatos en la web (O'Neill; Lavoie; Bennett, 2003), la aplicación de este modelo de análisis sería perfectamente aplicable sobre un gran porcentaje de la red.

Para vectorizar la muestra documental se programó un script en lenguaje $p h p$ que automatiza la indización. De esta forma se genera un número de vectores $n$, siendo $n$ el número de palabras clave diferentes encontradas en el conjunto documental. Los vectores tendrán tantos componentes como número de documentos en los que aparezca cada palabra clave. Es decir, siendo $X=\left\{x_{1}, \ldots, x_{m}\right\}$ el conjunto de documentos de la muestra, e $Y=\left\{y_{1}, \ldots, y_{n}\right\}$ el conjunto de palabras clave, la matriz $R \subseteq X \times Y$ representaría las relaciones de correspondencia binaria entre $X$ e $Y$.
En la muestra documental estudiada, formada por 269 documentos $(m=269)$, se encontraron 2.161 palabras clave diferentes $(n=2.161)$. Con el objetivo de que puedan ser empleadas para la recuperación de información, en este modelo son consideradas como categorías en sí mismas, y cada aparición de una de ellas en la descripción de un documento será considerada como una relación de pertenencia de éste a la categoría.

Dado que el número de categorías es muy amplio, para aumentar la cobertura de cada una (contabilizada en número de documentos) se propone un procedimiento que calcule el grado de pertenencia entre documento y categoría teniendo en cuenta más variables que la simple aparición de la palabra clave en la descripción del documento.

El funcionamiento de este procedimiento se basa en una serie de condicionales que determinarán el grado de pertenencia entre documento y categoría $G P(x, y)$ (siendo $x$ el documento e $y$ la categoría) de la siguiente forma:

- Si en el documento $x$ apareciera la palabra clave o categoría $y$, el $G P(x, y)$ tomará un valor igual a 0,5 (igual a 0 en caso contrario), sin perjuicio de que en los siguientes condicionales pueda incrementarse.

- Si en el título del documento $x$ apareciera la palabra clave $y$, el $G P(x, y)$ determinado en el primer condicional se incrementaría en 0,5 no teniéndose en cuenta los siguientes condicionales.

- Si en el resumen del documento $x$ apareciera la palabra clave $y$, el $G P(x, y)$ determinado en el primer condicional se incrementaría en 0,25 , y no se tendrían en cuenta los siguientes condicionales.

- Si en el cuerpo del documento $x$ apareciera la palabra clave $y$, el $G P(x, y)$ determinado en el primer condicional se incrementaría en 0,125 .

Tras aplicar esta serie de condicionales se obtiene una matriz $G P=\left\langle X \times Y, G P_{x y}\right\rangle$, donde a diferencia de la matriz $R$, las relaciones entre categorías y documentos ya no se representan de forma binaria, sino de forma ponderada con valores que oscilan entre 0 y 1 , siendo 0 un $G P(x, y)$ nulo, mientras que 1 sería el mayor $G P(x, y)$ posible.

Sobre esta matriz se realiza un ajuste de los grados de pertenencia de acuerdo con la siguiente premisa: cuanto mayor número de relaciones de pertenencia a categorías diferentes tenga un documento, menor debería ser su grado de pertenencia hacia cada una de estas categorías. Este ajuste se obtiene mediante la fórmula 1. En ella $G P(x, y)$ es el grado de pertenencia del documento $x$ a la categoría $y$; por su parte $N C(x)$ es el 
número de categorías diferentes a las que pertenece el documento $x$.

$$
G P(x, y)_{a j u s t a d o}=\frac{G P(x, y)}{N C(x)}
$$

Fórmula 1

Una vez realizado el ajuste se procede a calcular el grado de representatividad de cada categoría, con el objetivo de utilizar esa variable como criterio para la elección de las 100 categorías finales que conformarán la representación visual perseguida.

A diferencia de la cobertura de cada categoría, que como indicábamos con anterioridad sería cuantificable por el número de documentos que presentasen algún grado de pertenencia hacia ella, el grado de representatividad de cada una $(G R)$ debería tener en cuenta, además, la suma de los grados de pertenencias entre sus documentos y el total de categorías a las que pertenecen. La razón de este procedimiento se fundamenta en que no sólo de debe 'premiar' a la categoría por número de documentos representados, sino también por el grado de dificultad para que esos documentos sean representados por otras categorías. Finalmente, la función con la que se calcula el $G R$ es una adaptación de la fórmula de pesos $t f$, $i d f$ (fórmula 2) y en donde:

$$
G R\left(y_{z}\right)=\sum_{i=1}^{i=m}\left(\frac{G P\left(x_{i}, y_{z}\right)}{\sum_{r=1}^{r=n} G P\left(x_{i}, y_{r}\right)}\right)
$$

Fórmula 2

- GR(y) es el grado de representatividad de $y$.

- GP(x,y) es el grado de pertenencia de $x$ a $y$.

$-y$ es un elemento del conjunto de categorías $Y$.

$-x$ es un elemento del conjunto de documentos $X$.

$-m$ es el número total de documentos.

— $n$ es el número total de categorías.

Se probaron diversas fórmulas, incluso algunas más complejas. Sin embargo ésta fue la que mejores resultados dio en términos de cobertura y capacidad de inclusión de documentos para la muestra documental estudiada.

En función del cálculo del $G R(y)$ para cada categoría, se seleccionan las 100 categorías más representativas del conjunto documental, que podemos ver ordenadas según su grado de representatividad en el anexo I.
Anexo I

Lista ordenada de las categorías más representativas

Web design ................................................0,9792

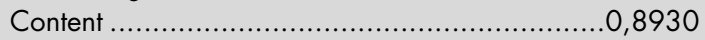

Search ….......................................... 0,8573

Pages .............................................. 0,7863

Email ...................................................... 0,7564

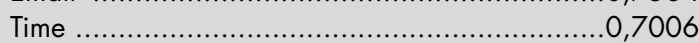

Bandwidth ................................................... 6618

Web usability................................................,6011

Messages ................................................0,5931

Advertising .......................................... 0,5869

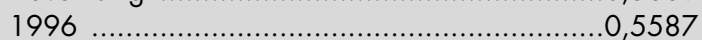

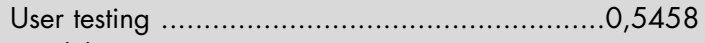

Guidelines ........................................... 0,5414

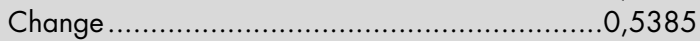

E-commerce ............................................. 0,5380

Browsers ........................................... 0,5332

History .............................................. 0,5330

Hypertext...................................................... 5211

Micropayments............................................... 0,5165

Designing …......................................... 0,5130

Link ...................................................... 0,4985

Slate redesign ........................................ 0,4959

Wap .......................................................,4921

Data ......................................................... 0,4878

Information architecture ............................,4862

Linking .............................................,4852

Animation ............................................ 0,4792

Software ................................................ 0,4733

Scrolling ................................................... 0,4409

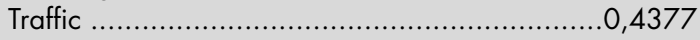

International usability ................................. 0,4363

Home page ........................................... 0,4332

Sales....................................................... 0,4260

Intranet .................................................. 0,4166

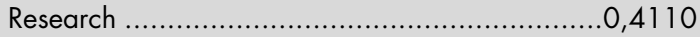

Internationalization ..................................... 0,4099

Security ................................................. 0,4035

Amazon.com ............................................... 0,4026

Pay .............................................................

Intranets ................................................ 0,4006

Mobile internet access ................................ 0,3995

Engagement........................................... 0,3945

Browser navigation feature ..........................0,3929

Devices ..................................................... 0,3905

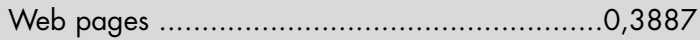

Standards ............................................. 0,3864

Structure .......................................... 0,3832

Writing ................................................. 0,3821

Predictions .......................................... 0, 3797

Feedback ......................................... 0,3780

Examples........................................... 0, 3717

Www ............................................... 0,3692

Surveys .................................................... 0,3666

Zipf distribution ...................................... 0,3648

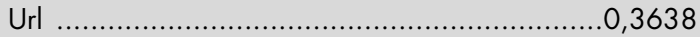

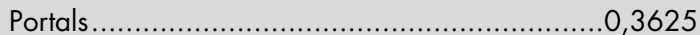

Pdf ..................................................... 0,3594

Multimedia ...............................................0,3569

Video …................................................ 0,3545

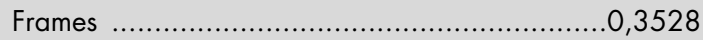

Disabilities ........................................... 0,3526

Intranet portals ....................................... 0,3516

Response time ......................................... 0,3502

Linkrot ............................................... 0,3492

Customization ....................................... 0,3456 


\section{LA NUEVA REVIS『A \\ SOBRE EL MUNDO DE LAS BIBLIOTECAS}

CBibliot Giblio
Borse: Whas

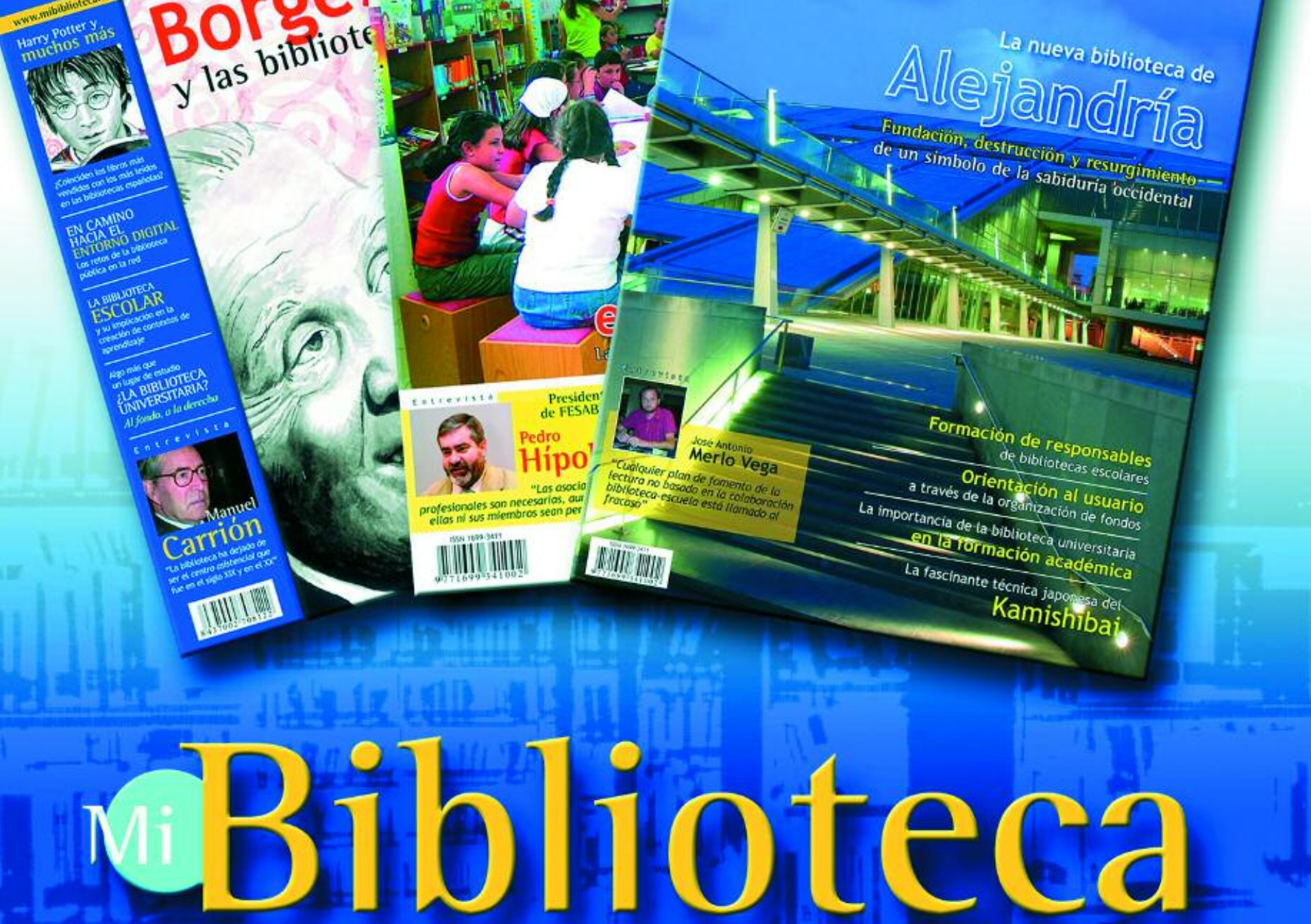

La revista del mundo bibliotecario

\section{Suscríbete.}




\begin{tabular}{|c|c|c|}
\hline 66 & State ....... & $.0,3455$ \\
\hline 67 & Archives ............. & $.0,3452$ \\
\hline 68 & Microtransactions. & $.0,3450$ \\
\hline 69 & Future $\ldots \ldots \ldots \ldots \ldots \ldots$ & $.0,3437$ \\
\hline 70 & Clickthrough ....... & $.0,3419$ \\
\hline 71 & $1997 \ldots \ldots \ldots \ldots$ & $.0,3372$ \\
\hline 72 & Mobile devices..... & 0,3370 \\
\hline 73 & Outsourcing ............ & $.0,3318$ \\
\hline 74 & Usability guideline & $.0,3308$ \\
\hline 75 & Bass curve ................ & $.0,3273$ \\
\hline 76 & TV ......................... & $.0,3273$ \\
\hline 77 & Ecommerce $\ldots \ldots \ldots \ldots$ & $.0,3246$ \\
\hline 78 & Size ........... & 0,3244 \\
\hline 79 & Monitor ........ & $.0,3241$ \\
\hline 80 & Text.................... & $.0,3230$ \\
\hline 81 & Bookmarks............. & $.0,3225$ \\
\hline 82 & Mobile internet....... & $.0,3186$ \\
\hline 83 & Hypertext links ...... & $.0,3176$ \\
\hline 84 & Media ................... & $.0,3167$ \\
\hline 85 & $1995 \ldots \ldots$ & $.0,3162$ \\
\hline 86 & $1999 \ldots \ldots \ldots$ & $.0,3159$ \\
\hline 87 & Europe ................. & $.0,3150$ \\
\hline 88 & Windows............. & $.0,3147$ \\
\hline 89 & Recruiting ............. & $.0,3146$ \\
\hline 90 & Affiliates programs & $.0,3135$ \\
\hline 91 & Computers .............. & $.0,3112$ \\
\hline 92 & Increasing returns .. & $.0,3110$ \\
\hline 93 & Reach .................... & $.0,3099$ \\
\hline 94 & Roi .............. & 0,3066 \\
\hline 95 & Publishing . & $.0,3063$ \\
\hline 96 & Staff ........... & $.0,3042$ \\
\hline 97 & $\mathrm{Html} \ldots \ldots \ldots$ & 0,3031 \\
\hline 98 & Redesign ............... & $.0,2989$ \\
\hline 99 & Preference .............. & $.0,2982$ \\
\hline 100 & Television .............. & $.0,2974$ \\
\hline
\end{tabular}

Por último se comprueba que todos los documentos de la muestra pertenecen al menos a alguna de las categorías finalmente seleccionadas. De hecho, como hemos visto, el éxito de la función para la elección de las categorías finales viene dado por su capacidad para determinar cuáles son las más inclusivas (cobertura y representatividad).

\subsection{Reducción de la dimensión}

Con el objetivo de representar visualmente el conjunto de categorías seleccionadas y sus relaciones en un espacio bidimensional, se aplicó la técnica de reducción dimensional $M D S$, que ha demostrado ampliamente su validez como herramienta de visualización (McQuaid, et al.; 1999).

Para ello se utilizó el paquete estadístico SPSS, al que se alimentó con la matriz de grados de pertenencia entre documentos y categorías que habíamos generado en la etapa anterior. Para calcular la matriz de (di)similaridades entre categorías se utilizó la función de correlación $\rho$ de Pearson. La aplicación de la técnica $M D S$ sobre esta matriz de distancias entre categorías, nos ofrece como resultado las posiciones o coordenadas en un espacio bi-dimensional para las diferentes categorías.
Para su implementación en un prototipo funcional consultable vía web, se representaron visualmente los rótulos de cada categoría en dichas posiciones o coordenadas mediante el lenguaje de etiquetado $S V G$ ( $s c a$ lable vector graphics) (Ferraiolo; Jun; Jackson, 2003).

\subsection{Interactividad y efecto ojo de pez}

Una vez que se genera la representación visual en forma de agrupaciones de categorías es necesario dotarla de cierta interactividad para que pueda funcionar como interfaz de recuperación de información. Su funcionamiento consiste básicamente en que cuando el usuario hace clic sobre el rótulo de una categoría, obtiene como resultado una lista de aquellos documentos pertenecientes a ella, ordenados por su grado de pertenencia.

Para ello la interfaz principal invoca a otro script en lenguaje $p h p$ que genera la lista de resultados, mostrando para cada documento su título, resumen y grado de pertenencia a la categoría en forma de porcentaje. Al hacer clic sobre el título de cada documento presentado el navegador se redirigirá al url correspondiente.

\section{«Cuando el usuario no tiene claro qué está buscando requiere sistemas alternativos que le permitan reconocer visualmente la información buscada y el modo de acceder a ella»}

A la interfaz principal se le ha provisto de un mecanismo de distorsión visual basado en el efecto ojo de pez, originalmente propuesto por Furnas (1986), con el objetivo de facilitar la exploración visual del conjunto de categorías. Es una técnica tipo Focus+context, que consiste en distorsionar una representación visual haciendo más visible el foco y minimizando la zona visual contextual o periférica (Herrero-Solana; Hassan-Montero, 2006). Furnas (1986) fue quien lo formalizó a través de su función de grado de interés o DOI (degree of interest), que asigna a cada elemento visual un valor que representa el interés del usuario en visualizarlo (fórmula 3).

$$
\begin{gathered}
\operatorname{DOI}(x, y)=A P I(x)-D(x, y) \\
\text { Fórmula } 3
\end{gathered}
$$

$\operatorname{DOI}(x, y)$ es el grado de interés que para el usuario tiene $x$, cuando el elemento foco que se está visualizando es $y$. API $(x)$ es el valor global de la importancia 


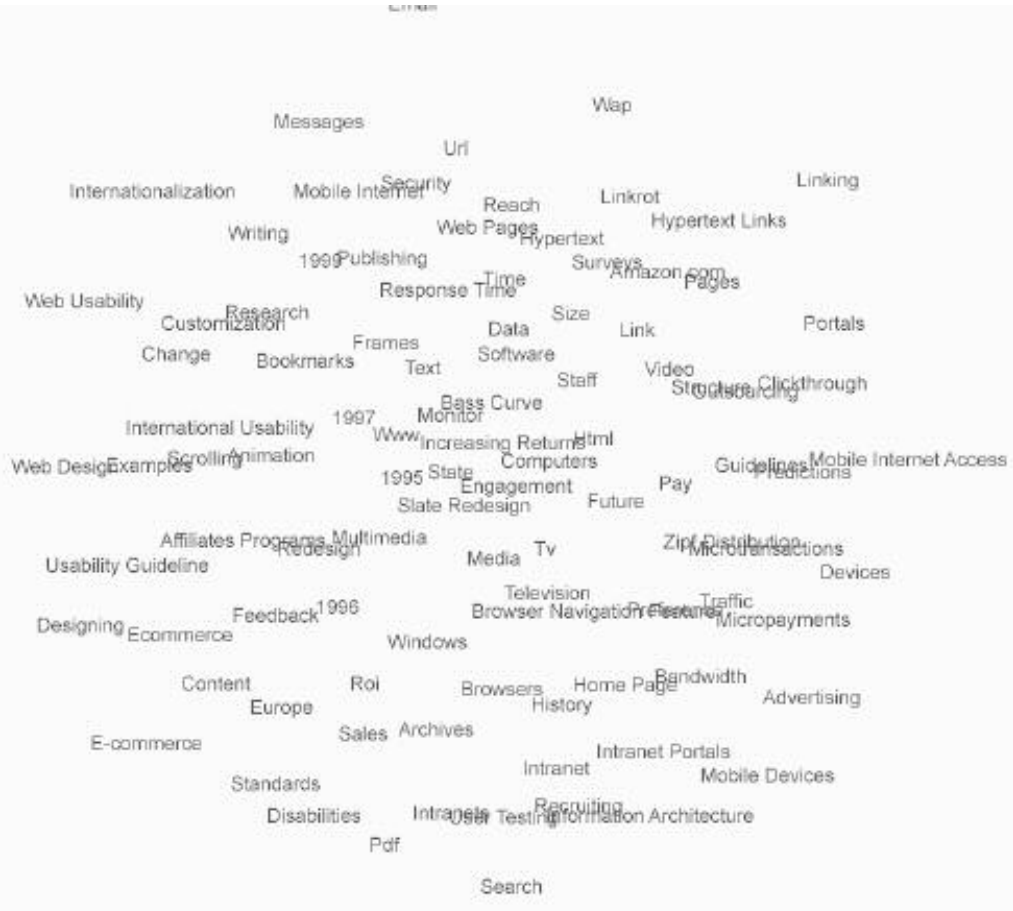

Figura 1. Interfaz principal del prototipo desarrollado

a priori (a priori importance) que tiene el elemento $\mathrm{x}$, $y D(x, y)$ la distancia existente entre el elemento $x$ y el elemento focal $y$.

Lo que se pretende es que cuando el usuario sobrevuele con el puntero el rótulo de una categoría (elemento focal), como consecuencia se produzca una transformación del tamaño y posición de los rótulos de las categorías. El rótulo de la categoría focal sería el que adquiriera mayor tamaño, mientras que el del resto de rótulos se calcularía en una relación inversamente proporcional a su distancia con respecto a la categoría focal, es decir, a más distancia menor tamaño.

Con relación a las posiciones, mientras que el elemento focal no cambia de posición, las del resto de categorías variarán alejándose de manera inversamente proporcional a su distancia original frente al elemento focal. Este desplazamiento está justificado porque, en caso contrario, el aumento de los tamaños de los rótulos más cercanos al elemento focal produciría un solapamiento entre rótulos que impediría su lectura.

El mecanismo aquí propuesto es una adaptación de la función original $D O I$, donde el grado de interés del elemento $x$ cuando el elemento focal es $y$ se corresponde con la distancia euclídea entre $x$ e $y$, dado que en una representación $M D S$ esta distancia indica el grado de similaridad entre elementos (fórmula 4).

$$
\operatorname{DOI}(x, y)=D(x, y)
$$

Fórmula 4
La transición entre vista original y vista distorsionada, de cara al usuario se realiza en forma de animación mediante una transformación progresiva de tamaños y posiciones. Como indican Lamping, Rao y Pirolli (1995), las transiciones animadas ayudan al usuario a asimilar los cambios entre vistas. Además, este comportamiento visual favorecerá minimizar el problema de la preservación del mapa mental que el usuario adquiere en la visualización previa a la distorsión (Herrero-Solana; Hassan-Montero, 2006).

\section{Resultados}

El aspecto final de la interfaz podemos apreciarlo en la figura 1. Cuando el usuario se ayuda del ratón en su exploración visual, sobrevolando con el puntero el rótulo de una categoría (elemento focal), se produce la distorsión visual de ojo de pez. Se puede observar en la serie de capturas de pantalla de la figura 2, donde el elemento focal sobrevolado es la categoría roi (return of investment).

Una vez que el usuario hace clic sobre una categoría, el sistema le ofrece como resultado una lista con los documentos ordenados por su grado de pertenencia, facilitando además un mecanismo de regreso a la interfaz principal en forma de enlace (map index), como podemos observar en la figura 3 .

\section{Evaluación}

En la literatura científica sobre visualización de información, en concreto la orientada al diseño de VIRI, 

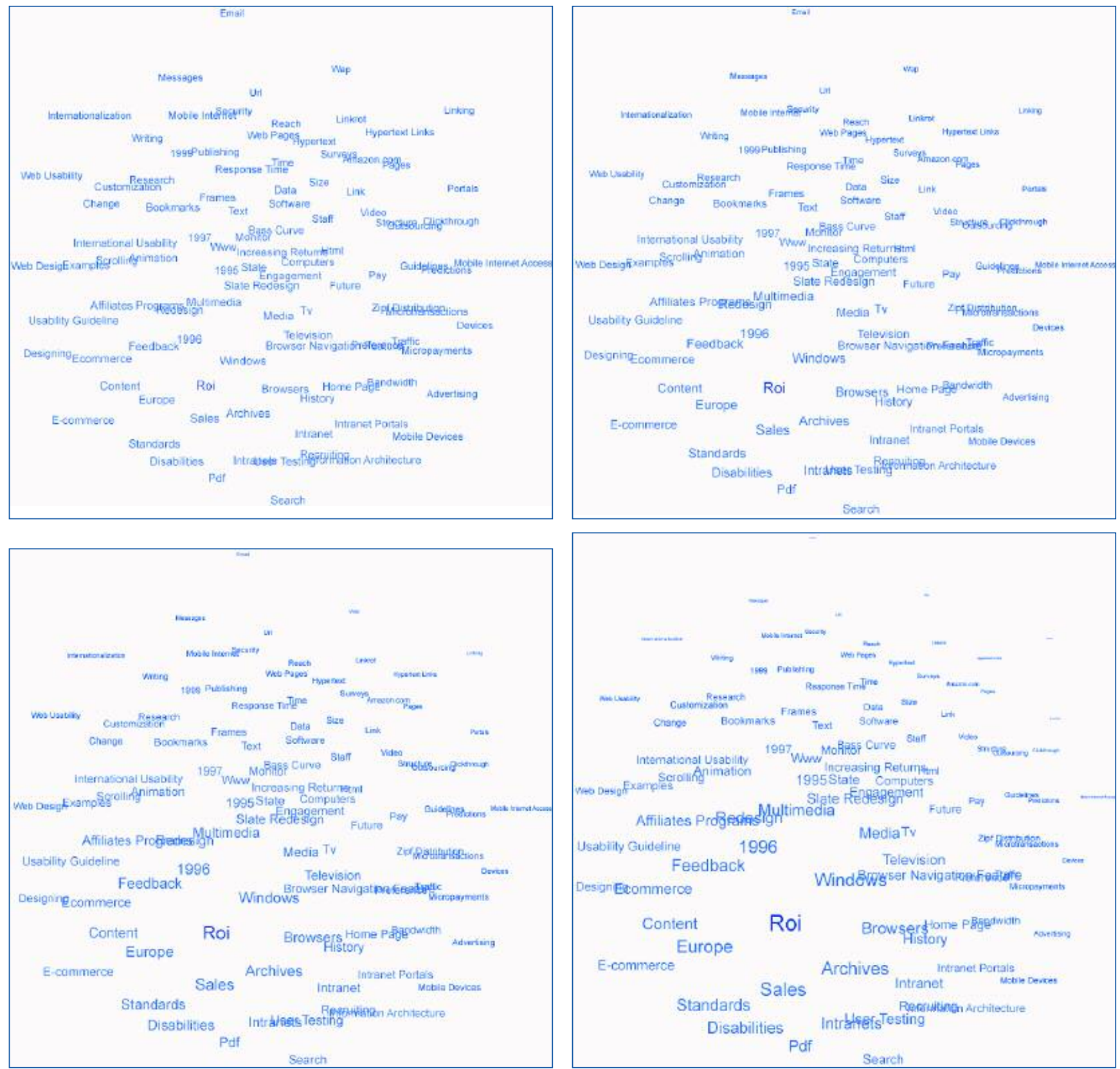

Figura 2. Transición de vista original a vista distorsionada en el prototipo desarrollado

es muy frecuente encontrar trabajos en los que las interfaces o propuestas de modelos no se evalúan ni validan de forma alguna. A pesar de que esta situación parece estar cambiando, lo hace lentamente y en casos todavía muy esporádicos (Chen; Czerwinski, 2000).

En el presente trabajo se ha realizado un estudio de evaluación preliminar para descubrir carencias, fallos y posibles mejoras del modelo de interfaz propuesto. La metodología de evaluación empleada ha sido el test basado en usuarios reales y el análisis cualitativo de los resultados. Se pretende de esta forma determinar su usabilidad y la del modelo de aplicación del efecto de ojo de pez empleado.

\section{a. Participantes y preparación}

Se contó con cinco hombres y cinco mujeres, estudiantes del programa de doctorado de documentación

KAP INDEX $=R O$

\section{RESULTS (5 documents)}

Do Productivity Increases Generate Economic Gains?

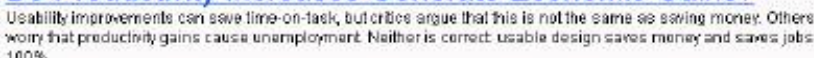

Return on Investment

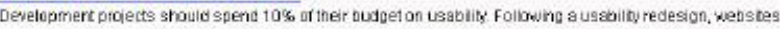

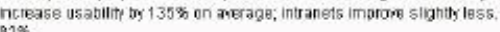

Intranet Usability: The Trillion-Dollar Question

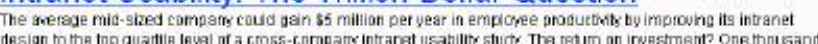
bercentor more.

Intranet Portal Usability and Design

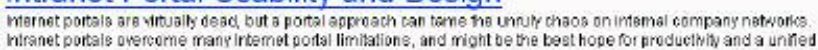
user esperiance
$57 \%$

Paper Prototyping: Get User Data Before You Code

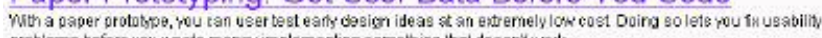

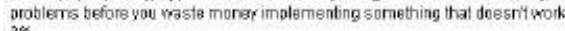

Figura 3. Resultados para la categoría 'ROI' en el prototipo desarrollado 
The second mind fusion fest

La emoción de conectar mentes abiertas

Un acto pensado desde la idea de que es preciso pensar hoy como en el Renacimiento: la mezcla de empresa, ciencia y arte, para definir nuevo valor económico. Desde la emoción de conectar mentes abiertas. Una mind fusion fest.

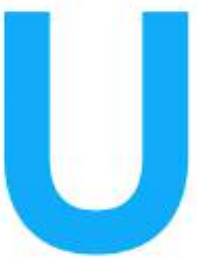

na ocasión única para escuchar, aprender, descubrir, y conectar, dirigida a profesionales de la empresa, la ciencia, y las artes con un factor común: la curiosidad por las ideas y las cosas, y la voluntad de transformar la sorpresa en nuevo valor económico. Un acontecimiento único en Europa, que une la fuerza de la creatividad con las emociones. Desde la convicción de que es preciso un nuevo Renacimiento en las organizaciones: hay que atreverse a acelerar el futuro, hoy.

\section{6-07 Julio}

Hotel Ra *****

Beach Thalasso SPA

El Vendrell

Patrocinan:

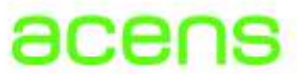

\section{ALTRAN}

\section{A QUIÉN VA DIRIGIDO...}

A 100 personas (muy especiales) que desean dar nueva energía a sus vidas y profesiones a través de la sintonía con ideas y personas diferente, a profesionales procedentes de campos distintos, que creen en el poder de la imaginación como nuevo factor decisivo en los negocios; a personas con energía para transformar su entorno y que quieren compartir sus inquietudes y explicar en qué están innovando ahora.

\section{LOS PONENTES...}

Profesionales que están redefiniendo los negocios de hoy, que tienen algo que decir sobre lo que está pasando en el mundo, que piensan y actúan de manera diferente, que replantean el momento de la empresa, que son

Un acto emocio-intelecutal pensado y conducido por Alfons Cornella y el equipo Infonomia.

Ideas, experiencias, personas...

Todo en reNacer 2006.

¿Te lo vas a perder?

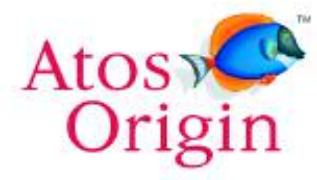

node

innovation for growth

\section{Telefonica}

\section{$\nabla$ infonomia \\ LARED DE INNOVADORES}

\section{www.infonomia.com/renacer06}


e información científica del Departamento de Biblioteconomía y Documentación de la Universidad de Granada. Como paso previo a la conducción del test se les impartió una sesión preparatoria en la que se explicaron los principios básicos de la recuperación de información por browsing y se describió la interfaz y su funcionamiento, haciendo especial hincapié en el efecto visual de ojo de pez.

\section{b. Tareas y procedimiento}

La prueba fue realizada por todos los participantes de forma individual con el fin de poder observar su comportamiento en la interacción, su reacción ante el uso de la interfaz, así como también las dudas que se planteaban durante la realización de las tareas.

La labor encomendada consistió en la recuperación de dos documentos, uno de ellos mediante la interfaz propuesta en este trabajo, y el otro mediante la misma interfaz pero con el efecto de ojo de pez desactivado.

Los dos documentos a localizar, que eran los mismos para todos los participantes, extraídos del conjunto documental de forma aleatoria, se les entregaban sin título ni resumen antes de comenzar el test. A cada uno se le dio el tiempo que estimara necesario para su lectura y comprensión para que posteriormente procedieran a realizar la búsqueda. En la mitad de los casos tuvieron que buscar el primer documento mediante la interfaz con ojo de pez (figura 2), y el segundo documento a través de la otra (figura 1). El resto de los participantes lo hicieron de forma inversa.

\section{«El efecto de ojo de pez es una técnica tipo focus+context que consiste en distorsionar una representación visual haciendo más visible el foco y minimizando la zona visual contextual o periférica»}

Para cada tarea se contabilizó el tiempo que cada usuario tardó en encontrar el documento, además de analizar su comportamiento frente a la interfaz, los errores que cometió, y las estrategias utilizadas en la búsqueda. Al finalizar se les pidió que comentaran cómo había sido su experiencia con ambas interfaces.

\section{c. Resultados e interpretación}

Los tiempos empleados en la realización de la búsqueda no muestran diferencias significativas entre ambos casos, lo que sugiere que el empleo de la técnica de ojo de pez no parece influir en la eficiencia de la interacción. Un fenómeno observado durante la prueba
¿Recibes ya EPI en casa?

Por sólo 75 euros + 4\% IVA

puedes tener todos los meses

tu copia particular y leerla có-

modamente cuando quieras.

es que los participantes utilizaron estrategias de exploración y navegación muy diferentes, condicionadas por la existencia o no del efecto de ojo de pez. Cuando se encontraban frente a la interfaz con dicho efecto, dedicaban más tiempo a la exploración visual y, según se observó, sólo seleccionaban una categoría para ver sus resultados cuando tenían cierta seguridad de que ésa era la categoría adecuada. La razón de este comportamiento puede deberse, en parte, al carácter interactivo del efecto visual y la distracción que puede ocasionar la animación; pero también, a que una vez resaltadas ciertas categorías, se disminuye la sobrecarga visual siendo más fácil explorar visualmente la interfaz y tomar decisiones sobre qué categoría puede resultar más afín temáticamente al documento buscado.

Cuando se encontraban frente a la interfaz desprovista del efecto de ojo de pez, realizaban una navegación más compulsiva y poco meditada, es decir, una navegación por ensayo y error, en la que hacían clic con mayor frecuencia en las categorías para observar qué documentos se mostraban. Una posible interpretación es que el número elevado de categorías presentes provoque sobrecarga visual y el usuario prefiera probar con la primera categoría que crea puede llevarle a su objetivo, en vez de explorar más detenidamente la interfaz.

Como resultado de este comportamiento los usuarios solían cometer más errores (entendiendo éstos como la exploración de categorías incorrectas) cuando usaban la interfaz sin ojo de pez. Este comportamiento parece demostrar que el efecto ojo de pez propuesto mejora la eficacia en la consecución de tareas de browsing aplicadas a la recuperación de información.

Al finalizar la prueba los usuarios expresaron su grado de satisfacción en el uso de las dos interfaces, valorando de forma mucho más positiva el empleo de la interfaz con ojo de pez. Tan solo uno la valoró de forma negativa, alegando que la animación de los rótulos de las categorías le producía un cierto mareo. Por su parte, en la mayoría de los casos valoraron como muy útil o útil la interfaz con ojo de pez. 


\section{Conclusiones y trabajos futuros}

En el presente trabajo se ha propuesto un modelo de interfaz mediante una combinación metodológica hasta la fecha no estudiada en la literatura científica: análisis de meta-contenido, escalamiento multidimensional o MDS, y efecto de distorsión visual de ojo de pez, y se ha desarrollado un prototipo funcional.

Los resultados sugieren que el modelo propuesto para realizar el análisis de meta-contenido resulta válido tanto para determinar el grado en el que cada documento se encuentra representado por una palabra clave o categoría, como para determinar cuál es el grupo de categorías más representativas del conjunto documental.

Otro aspecto importante a señalar es la utilidad del $M D S$ como técnica de clasificación y distribución visual. Como indicábamos, su elección se basó en la validez demostrada como herramienta de visualización y tras la evaluación con usuarios se ha comprobado que éstos no han encontrado problemas para comprender la metáfora visual resultante de su aplicación. Sin embargo, la técnica de $M D S$, por su carácter iterativo, tiene el inconveniente de que a mayor número de dimensiones originales a reducir, mayor tiempo de procesamiento. Por esta razón, esta técnica no resultaría viable en la generación dinámica de representaciones visuales para conjuntos documentales de gran tamaño.

Respecto al efecto ojo de pez, si nos atenemos a los resultados del test con usuarios, podemos concluir que la propuesta de aplicación supone, en términos de eficacia y satisfacción de uso, una mejora significativa para la interfaz. Aun así creemos que, en próximos trabajos, sería necesario evaluarla con mayor número de participantes y perfiles más heterogéneos, para comprobar si los resultados corroboran los obtenidos en la presente evaluación.

Además, la propuesta de aplicación de ojo de pez creemos sería susceptible de mejoras. En concreto, ésta no hace uso de la variable API (a priori importan$c e$ ), que en el trabajo original de Furnas (1986) se calculaba en base al nivel jerárquico de cada elemento. En estructuras de datos multidimensionales donde no existen relaciones de jerarquía, Chen (1998) propone su cálculo según el número de accesos o visitas de usuario recibidas para cada elemento, considerando que es un buen indicador de su importancia. En el contexto de este trabajo una posible opción sería determinar el API de cada categoría basándose en el número de sus documentos representados. Esta opción, así como otras posibles, deberán ser consideradas y estudiadas en futuros trabajos.

\section{Bibliografía}

Baeza-Yates, R. «Excavando la web». En: El profesional de la información, 2004, enero-febrero, v. 13, n. 1, pp. 4-10.

Börner, K.; Chen, C.; Boyak, K. W. «Visualizing knowledge domains». En: Annual review of information science and technology, 2003, n. 37, pp. 179-255.

Chen, C. «Generalised similarity analysis and pathfinder network scaling». En: Interacting with computers, 1998, n. 10, pp. 107-128.

Chen, C.; Czerwinski, M. P. (2000). «Empirical evaluation of information visualizations: an introduction». En: International journal of human-computer studies, 2000, n. 53, pp. 631-635.

Ferraiolo, J.; Jun, F.; Jackson, D. Scalable vector graphics (SVG) 1.1 Specification. W3C Recommendation 14 January 2003. http://www.w3.org/TR/2003/REC-SVG11-20030114/

Furnas, G. W. «Generalized fisheye views». En: Mantei, Marilyn; Orbeton, Peter (eds.). Proceedings of the Sigchi conference on human factors in computing systems, 1986, pp. 16-23.

Hartigan, J. A. Clustering algorithms. New York: John Wiley \& Sons, 1975 .

Herrero-Solana, V. Modelos de representación visual de la información bibliográfica: aproximaciones multivariantes y conexionistas. Tesis doctoral. Granada: Universidad, Departamento de Biblioteconomía y Documentación, 2000.

Herrero-Solana, V.; Hassan-Montero, Y. «Metodologías para el desarrollo de interfaces visuales de recuperación de información: análisis y comparación». En: Information research, 2006, April, v. 11, n. 3. http://informationr.net/ir/11-3/paper258.html

Kamada, T.; Kawai, S. «An algorithm for drawing general undirected graphs». En: Information processing letters, 1989, n. 31, pp. 7-15.

Kohonen, T. Self-organization and associative memory. 3rd Edition. Berlin: Springer-Verlag, 1989.

Kruskal, J. B.; Wish, M. Multidimensional scaling (quantitative applications in the social sciences). Newbury Park: SAGE, 1978.

Lamping, J.; Rao, R.; Pirolli, P. «A focus+context technique based on hyperbolic geometry for visualizing large hierarchies». En: Proceedings of the ACM Sigchi conference on human factors in computing systems. New York: ACM Press, 1995, pp. 401-408.

Lin, X. «Map displays for information retrieval». En: Journal of the American Society for Information Science, 1997, v. 48, n. 1, pp. 40-54.

Marchionini, G. Information seeking in electronic environments. New York: Cambridge University Press, 1995.

Marcos-Mora, M. C. Interacción en interfaces de recuperación de información: conceptos, metáforas y visualización. Gijón: Ediciones TREA, 2004.

McQuaid, M. J., et al. «Multidimensional scaling for group memory visualization». En: Decision support systems, 1999, n. 27, pp. 163-176.

O'Neill, E. T.; Lavoie, B. F.; Bennet, R. «Trends in the evolution of the public web». En: D-lib magazine, 2003, April, v. 9, n. 4.

http://www.dlib.org/dlib/april03/lavoie/04lavoie.html

Salton, G. Automatic text processing: the transformation, analysis and retrieval of information by computer. Reading, MA: Addison Wesley, 1989.

Schvaneveldt, R. (ed.). Pathfinder associative networks: studies in knowledge organization. Norwood, NJ: Ablex, 1990.

\author{
Yusef Hassan-Montero y Víctor Herrero-Solana, Grupo \\ SCImago, Granada. \\ yusefhassan@gmail.com \\ victorhs@ugr.es
}

